\title{
Agenda y particularización del discurso político chino: un análisis evolutivo en la guerra comercial con los Estados Unidos
}

\author{
MARIANO MOSQUERA*
}

\begin{abstract}
RESUMEN
¿Qué tendencias pueden identificarse en un análisis evolutivo sobre la agenda china en la guerra comercial con los Estados Unidos? La investigación analiza los dos documentos oficiales en la materia del Gobierno de la República Popular China (de septiembre de 2018 y de junio de 2019), desde un marco teórico centrado en la racionalidad política china y en los estudios de agenda, y con herramientas propias del análisis autómatico de textos. Se obtiene como conclusión que el discurso político chino sostiene — como eje central — la intención de expandir el debate comercial a otros significados económicos, mantiene la crítica al proteccionismo y defiende su posición sobre la propiedad intelectual en China. Sin embargo, en el año 2019 en el discurso político chino adquieren una mayor centralidad los valores y principios cooperativos típicos de la retórica política china. Esto se destaca sobre la estategia china de deconstrucción del discurso occidental sobre el conflicto comercial, lo cual es predominante, con abundante particularización discursiva, en el año 2018.
\end{abstract}

Palabras clave

Agenda; discurso; evolución; guerra comercial; China.

\section{Tithe}

Agenda and particularization in Chinese political discourse: Evolutive analysis over trade war with United States

\section{EXTENDED Abstract}

Modern Chinese political discourse, regarding foreign policy, has aroused considerable interest in the academic world, both by Western intellectuals and by Chinese scholars. In both cases, this interest is found in the possibility of generating a kind of translation of the particular Chinese discursive logics towards significant standards for theWest. Furthermore, as an ultimate goal, it is hoped that this interpretive task may favor better political understandings between China and the West. In this sense, the current trade war between China and the United States represents a relevant object of study, to analyze from a Chinese political and discursive perspective.

In this research paper we ask: what trends can be identified in an evolutionary analysis of the Chinese agenda in the trade war with the United States? The research analyzes two official documents on the subject (White papers) from the highest echelons of the government of the People's Republic of China:The Facts and China's Position on China-US Trade Friction (September 2018) and China's Position on the China-US Economic and Trade Consultations (June 2019). These two documents represent the official vision of China and its evolution, in accordance with contextual changes.

The research develops a theoretical framework focused on Chinese political rationality and its peculiarities. To make this conceptual framework transparent, it is compared with western political rationality. Different dimensions are worked on: political inclusion of actors in decision-making processes, framework for political action, processes of political action, the logic to determine solutions, and time horizon of political action. In theWest, democratic horizontality grants argumentative flexibility, and stable frameworks for action restrict it. In China, institutional dynamism grants argumentative flexibility and the hierarchical and particular order restricts it. Democracy with stability versus hierarchy with change: both political schemes establish the argumentative limits. In other words, the dimensions of political rationality have a direct impact on the content and form of Chinese political discourse.

\section{DOI:}

https://doi.org// 0.15366/relacionesinternacionales2021.48.012

Formato de citación recomendado:

MOSQUERA, Mariano (202I).“Agenda y particularización del discurso político chino: un análisis evolutivo en la guerra comercial con los Estados Unidos”, Relaciones Internacionales, $n^{\circ}$ 48, pp. 23I-246.

\section{* Mariano} MOSQUERA, Director del Centro de Estudios La Franja y la Ruta. Contacto: mariano.mosquera@ ucc.edu.ar

Recibido:

10.07.2020

Aceptado:

21.11 .2020 
Agenda studies is used strategically in this research to analyze Chinese political discourse. The pragmatic use of language and the combination of Confucianism and Marxism in Chinese political discourse can be identified from the perspective of agenda studies. In other words, an agenda approach allows us to consider different dimensions of Chinese political rationality and rhetoric: hierarchy established in a text; non-linearity of the solutions agenda; enabling, contradictory and strategic frameworks; particular context and its relation to general context; and finally, dynamism of the information content.

The two specific research questions in this article are: How does China define and particularize its agenda in the trade war with the United States? What trends can be identified in an evolutionary analysis over this agenda? Our research hypothesis is: In Chinese official documents on the trade war with the United States, there are trends regarding political agenda, particularistic resources and an articulation of both.

From a methodological point of view, the emergence of new technologies has allowed for the expansion of the frontiers of textual analysis. The present work uses cutting edge automatic natural language processing tools to study the Chinese agenda, such as: identification of parts of speech (Part of Speech Tagging), construction of concordance matrices (with Key Word in Context framework), named entity recognition, and relationship vectors calculation. Also, comparative analysis techniques are used to analyze evolution of the PRC's agenda in the trade war with the United States.

In this way, the work proposes an original contribution (theoretical and empirical) to the field of social and political agenda studies, by adapting them to Chinese political rationality and discourse.

As conclusions, this investigation demonstrates the fulfillment of the hypothesis, since in the official Chinese documents related to the trade war with the United States there are tendencies on the political agenda, the particularistic resources and the articulation of both.

The document The Facts and China's Position on China-US Trade Friction expands the trade discussion towards industrial chains and investments. In this text, measures that correspond to national interests and those that affect the international community are listed, specifying the latter in the WTO arena. Also, goods and services of trade and their rates are particularized with data and proper names. Two other frameworks are relevant: the discussion on intellectual property, and the rules and regulations to foster cooperation. This second one (quite restricted) acts as an enabling framework, although it omits Chinese rhetoric of values and principles.

China's Position on the China-US Economic and Trade Consultations document maintains the trend of the central axis of the 2018 document, but it is less aggressive, by centralizing cooperation as an objective. The 2019 document does not offer as important a backing of authoritative resources as the 2018 document does. In addition, the trend of intellectual property debate in China remains very important in the 2019 document. However, as a relevant change, this second document presents a political agenda much closer to the contradictory and dialectical frameworks of China's classical Confucian and Marxist rhetoric (terms such as mutual benefit, mutual respect or win-win relationships are used).

There is a marked trend of important coincidences (expanding the discussion from commercial to economic, criticizing protectionism and holding a position in the debate on intellectual property in China), but the differences between documents are also relevant. In particular, the 2019 document embraces in a more friendly way the Chinese political rhetoric of cooperative values and principles, and avoids resources of authority. In this sense, less particularization implies lower levels of orientation.

From the point of view of our theoretical framework, both documents seek to highlight collective effectiveness (the development of a global community), and work on problem-solution logics. Furthermore, the change between the two documents reflects the dynamism in China's use of discursive resources.

In short, the second document of 2019 shows greater characteristics of a Chinese political discourse which encourages pragmatism. The first document of 2018 develops a more deconstructive position over the western rhetoric of the trade conflict, even using tools that are more typical of scientific discourse (with deductions or inductions from authoritative resources).

Chinese political discourse is dynamic, adapting to the context in a multiplicity of possible ways. Identifying continuities and changes in Chinese discourse from the theoretical understanding of its political rationality is essential to interpret current conflicts.

\section{KEYWORDS}

Agenda; discourse; evolution; trade war; China. 


\section{$\mathrm{I}$}

\section{ntroducción}

El presente trabajo tiene como objetivo desarrollar un análisis evolutivo sobre la agenda de la República Popular China en la guerra comercial con los Estados Unidos. Para alcanzar este objetivo se trabajó sobre los dos documentos oficiales chinos relativos a dicho conflicto y se analizó la definición y particularización de la agenda, así como sus tendencias en el tiempo. Se utilizan los documentos oficiales The Facts and China's Position on China-US Trade Friction (Los Hechos y la Posición de China en la Fricción Comercial China-Estados Unidos) de septiembre de 2018 y China's Position on the China-US Economic and Trade Consultations (La Posición de China en las Consultas Comerciales y Económicas China-Estados Unidos) de junio de 2019.

En esta investigación nos enfocamos en la visión oficial de China sobre la actual guerra comercial, que tiene sus inicios en la administración de Donald Trump. Sin embargo, una gran cantidad de estudiosos identifican "en la crisis financiera de 2008 un punto de inflexión sobre la rivalidad" (Zhao, 2019, p. 385). Esta crisis cambia "la naturaleza de la relación moderna entre estos dos países" (Tao, 2009, p. 28), generando una nueva era de competición. El "período de crisis post-financiera" (Zhao, 2013, p. 49) es el contexto histórico donde se inserta el presente trabajo y donde autores como Fan Wang (2008) resaltan una nueva relación de interdependencia competitiva entre Estados Unidos y China. Es importante destacar que la mayoría de los análisis sobre esta relación se producen desde enfoques realistas de las relaciones internacionales, como los artículos de Yuan (2010) y Xu (20I8), y sin estudios discursivos sobre la narrativa oficial china.

En el marco de este período de crisis post-financiera, la administración Trump realiza "el cambio de mayor alcance en la política hacia China desde 1972” (Li, 2019, p. 520), acentuando la rivalidad competitiva como conflicto explícito' y provocando el evento inédito que nos importa: la generación de un discurso oficial chino sobre la disputa moderna entre China y Estados Unidos. En este sentido, los dos documentos analizados representan casos de estudio únicos, por su carácter de white paper para el Gobierno de la República Popular China. Se trata, por lo tanto, de documentos de la más alta jerarquía que manifiestan una posición oficial cuidadosamente preparada por el gobierno central chino. Tanto por el marco teórico desarrollado como por la aplicación de una metodología acorde al objeto de investigación, esta investigación posee una marcada originalidad respecto a los antecedentes de estudio que se relevan en el primer apartado de este trabajo.

Las preguntas de investigación del presente artículo son dos: ¿de qué forma China define y particulariza su agenda en la guerra comercial con los Estados Unidos?, ¿qué tendencias pueden identificarse en un análisis evolutivo sobre dicha agenda? Nuestra hipótesis de investigación se estructura de la siguiente manera: en los documentos oficiales chinos sobre la guerra comercial con los Estados Unidos existen tendencias sobre la agenda política, los recursos particularistas utilizados y la articulación de ambos. Es importante aclarar que las tendencias reflejan las continuidades y, a su vez, dejan expuestos los cambios, como adaptaciones del discurso al contexto (Lima Bandeira y Rubaii, 20 I6; Eyal, 1980).

Los estudios de agenda se centran en la identificación de los temas y sus atributos que

Desde fines de 2016, durante la campaña presidencial de Trump, comienza la explicitación de la rivalidad como conflicto. Esta narrativa escala con las diversas medidas proteccionistas contra bienes chinos. 
influyen sobre las audiencias (Aruguete, 2015). Tanto por frecuencia de aparición como por activación en tándem (Gou, 20l4), esta identificación prescinde de observar las relaciones gramaticales del lenguaje. Este enfoque tiene un particular interés de aplicación sobre la retórica china, debido al uso pragmático del lenguaje, los modelos no-lineales de argumentación y, en definitiva, la combinación entre confucionismo y marxismo presente en el discurso político chino (Leonard, 2008). La preponderancia de ciertos términos (como conceptualización de sustantivos comunes de alta frecuencia), su relacionamiento y la particularización de determinadas narrativas - con diversos recursos de autoridad - juegan un papel importante y permiten responder a la primera pregunta de investigación. Por este motivo, el segundo capítulo de esta investigación trabaja en detalle el marco teórico que describe las particularidades discursivas del gigante asiático en su retórica oficial.Además, este marco conceptual justifica la aplicación del enfoque de agenda sobre el objeto de estudio.

Por otra parte, el surgimiento de nuevas tecnologías ha permitido expandir notablemente las fronteras del análisis de texto (Coulthard, 1996). El presente trabajo recupera el estado del arte en la materia y utiliza herramientas de procesamiento automático de lenguaje natural como: la identificación de partes del discurso (Part of Speech Tagging), el cálculo de matrices de concordancia (Key Word in Context), el reconocimiento de entidades (Named Entity Recognition) y la determinación de vectores de relacionamiento (Vylomova, Rimell, Cohn y Baldwin, 2016). Dado este contexto, el tercer capítulo desarrolla en profundidad la metodología utilizada. En este apartado se definen los criterios para la identificación de las tendencias, lo que permite responder a la segunda pregunta de investigación. El cuarto capítulo de este trabajo presenta los resultado de la investigación en dos partes. Una primera dedicada a la descripción estática y una segunda que analiza la evolución entre documentos. Finalmente se presentan las conclusiones del trabajo de investigación.

\section{I.Antecedentes}

El discurso político chino moderno, en lo que refiere a su política exterior, ha despertado un considerable interés en el mundo académico, tanto por intelectuales de Occidente como por estudiosos chinos (Xu, 20l4). En ambos casos, este interés se encuentra en la posibilidad de generar una especie de traducción de las particulares lógicas discursivas chinas hacia los estándares significativos para Occidente (Fairclough, 2003).Además, como fin último se espera que esta tarea interpretativa pueda favorecer mejores entendimientos políticos entre China y Occidente.

Juana Marisela Connelly (1991) ha estudiado la transición del discurso chino, desde la retórica revolucionaria hasta la conformación del discurso moderno, centrado en la paz y el desarrollo. En este primer antecedente de relevancia se destaca cómo los cambios ocurridos en diciembre de 1978 de la denominada Reforma y apertura china modificaron significativamente el discurso chino. Diversos hitos fueron incorporándose a este discurso moderno, como la base de los cinco principios de coexistencia pacífica de $1982^{2}$ que mantienen su vigencia en el discurso actual. En general, esta evolución del discurso político "se siguió afirmando en la posición de China

2 Se trata de los siguientes principios: I) Respeto mutuo a la soberanía e integridad territorial; 2) No agresión; 3) No intervención de un país en los asuntos internos de otro; 4) Igualdad y beneficio recíproco; 5) Coexistencia pacífica. 
como contraria al hegemonismo y defensora de los derechos de los paises del tercer mundo" (Conelly, I99I, p. 303).

El segundo antecedente de relevancia — para esta investigación - se trata de un estudio de Mariola Moncada Durruti (20II), donde la autora trabaja la influencia del confucionismo en el discurso actual del Partido Comunista Chino. Moncada Durruti (201I, p. 215) observa que es Jiang Zemin ${ }^{3}$ quien abre la puerta a una nueva inspiración en el discurso político chino sobre sus relaciones internacionales:

\begin{abstract}
“Jiang Zemin parafraseaba a Confucio y sugería la adopción del espíritu que encierra el aforismo 君子和而不同 como guía en las relaciones internacionales: buscar la armonía respetando las diferencias; asumir la diversidad cultural como oportunidad de enriquecimiento mutuo; armonizar sin igualar; mantener las diferencias culturales sin dar cabida al conflicto. Jiang ponía el énfasis en la palabra 'he' (armonizar), un concepto tradicional chino que debía traducirse, según Jiang, en lo político como respeto mutuo, en lo económico como reciprocidad y en lo cultural como conocimiento mutuo".
\end{abstract}

En este mismo sentido se han expresado autores occidentales clásicos como Daniel Bell (2008), quien destaca cómo el discurso político chino adopta oficialmente el confucionismo para dotar de valores y fundamentos morales al Gobierno y a sus acciones. También Mark Leonard (2008) trabaja sobre las ideas políticas chinas que incorporan un nuevo tipo de confucionismo (neoconfucionismo). Desde la perspectiva china, Li (2006, 20 I4) y Cheng (I99I) analizan el mismo fenómeno.

Por su parte, autores como Jiayu Wang (2017) y Guoxin Xing (2009) analizan la presencia de la ideología marxista en el discurso actual del gobierno de la República Popular China. Distintos recursos son identificados como adaptaciones modernas de la retórica revolucionaria. También, la investigación de Oviedo (20l4) refleja particularidades lógicas en el discurso chino y que son propias del método marxista, como lo son los usos de contradicciones para propiciar la síntesis dialéctica.

Un antecedente específico - desde el punto de vista de nuestro objeto de estudio- es el trabajo de Manuel de Jesús Rocha Pino (20I8). En esta investigación se analiza el discurso político oficial de China desde el año 2012 —desde el comienzo de la presidencia de Xi Jinping - y, en particular, aquel destinado a redefinir su relación con los Estados Unidos. Explica el autor que la resignificación discursiva es un nuevo "compromiso por parte del Gobierno de Xi jinping frente a Estados Unidos, para iniciar una relación políticamente simétrica, teniendo como prioridad crear vínculos de cooperación y mecanismos de construcción de confianza” (Rocha Pino, 2018, p. 2 I3).

Otros antecedentes relevantes son los trabajos de Zeng y Breslin (20l6), Wu (20I4), y Chase (20I2), quienes también analizan el discurso político chino que se encuentra dirigido

3 Ex Presidente de la República Popular China desde marzo de 1993 a marzo de 2003. 
especificamente hacia los Estados Unidos. Se dice:

"Xi enfatizaba cuatro áreas en las que ambos países podían reforzar sus relaciones de cooperación bilateral, I) entendimiento mutuo y confianza estratégica;2) respeto mutuo de los intereses centrales y las principales preocupaciones de cada parte; 3 ) mayor cooperación de beneficio mutuo; 4) más cooperación y coordinación en los asuntos internacionales y globales" (Chase, 2012, p. I3).

Los antecedentes seleccionados describen lógicas y caracterísiticas particulares del discurso político chino en el marco de sus relaciones internacionales, como: su orientación antihegemónica, la incorporación de valores confucianos y retórica marxista, y la opertavización de valores en forma de principios de cooperación internacional. Esto, incluso, se encuentra vinculado a los discursos dirigidos específicamente hacia los Estados Unidos. Además, Callahan (2016) sugiere que la combinación entre poder material y discurso es relevante en el contexto actual del crecimiento de China. Este autor nos dice que China "usa la fortaleza económica para construir una comunidad de destino común en Asia, algo que transformaría a China en un poder normativo generador de las normas del juego de la gobernanza global" (Callahan, 2016, p. 228). En este contexto, adquiere aún mayor relevancia el estudio del discurso político chino sobre sus relaciones internacionales.

\section{Marco teórico}

En este capítulo utilizamos la comparación con Occidente para describir las particularidades de la retórica política china. Esto es posible, solamente, al relacionar dicha retórica con los procesos de racionalidad en distintas dimensiones políticas clave (Wodak, 2009). El cuadro I describe esta comparación. 


\section{Cuadro I. Comparación de dimensiones sobre racionalidad y retórica política}

\begin{tabular}{|c|c|c|}
\hline Dimensión clave & Occidente & China \\
\hline $\begin{array}{l}\text { Fin del sistema } \\
\text { político }\end{array}$ & Gatantizar derechos individuales & Eficacia colectiva \\
\hline $\begin{array}{l}\text { Inclusión política } \\
\text { de actores en los } \\
\text { procesos de toma } \\
\text { de decisión }\end{array}$ & $\begin{array}{l}\text { Por democracia pluralista. Se trata de una } \\
\text { competencia entre actores en un marco } \\
\text { de igualdad formal (one man, one vote). } \\
\text { Los factores para ganar la competencia } \\
\text { son variables y desiguales, y son evaluados } \\
\text { de forma horizontal por la ciudadanía. } \\
\text { Es la evaluación horizontal la que } \\
\text { otorga flexibilidad para competir con } \\
\text { diferentes argumentos, haciendo difusos } \\
\text { los criterios de mérito (por argumentos } \\
\text { inconmensurables o por argumentos } \\
\text { generales que abarcan en exceso } \\
\text { múltiples diferencias) }\end{array}$ & $\begin{array}{c}\text { Por liderazgo. Se basa en la formación teórica } \\
\text { y práctica de los cuadros en contextos } \\
\text { institucionales particulares y dinámicos (no } \\
\text { generales y estables). } \\
\text { La ubicación en la estructura depende de } \\
\text { la evaluación sobre dicha formación que } \\
\text { realizan otros cuadros de una posición } \\
\text { superior. Es un esquema con un criterio } \\
\text { meritocrático, competitivo y jerárquico } \\
\text { en la evaluación. Es decir, el criterio de } \\
\text { formación en un contexto particular } \\
\text { reduce posibilidades de argumentación. Al } \\
\text { mismo tiempo, el dinamismo del contexto } \\
\text { institucional expande estas posibilidades. La } \\
\text { evaluación jerárquica considera ambas }\end{array}$ \\
\hline $\begin{array}{c}\text { Marco para la } \\
\text { actuación }\end{array}$ & $\begin{array}{c}\text { Rule of law. Los actores ganadores - } \\
\text { representantes- establecen leyes } \\
\text { generales —universales-y estables para } \\
\text { la actuación. } \\
\text { Los marcos favorecen la cooperación - } \\
\text { como otra cara de la competencia-y } \\
\text { la evaluación comparativa - reduce la } \\
\text { flexibilidad argumentativa- } \\
\text { Las fórmulas tienen una lógica de si } X \text { o } \\
\text { no } X \text {. Es un esquema que condiciona } a \\
\text { priori a los actores y sus acciones }\end{array}$ & $\begin{array}{c}\text { Rule by law. Se establecen a priori solamente } \\
\text { tres tipos de marcos: habilitantes, } \\
\text { contradictorios y en deconstrucción. } \\
\text { El primero con una fórmula lógica no-no } X \\
\text { (no prohibir). El segundo favorece la síntesis } \\
\text { dialéctica en los contextos particulares. } \\
\text { El tercero evidencia el proceso de crítica } \\
\text { estratégica —afirmación y crítica al mismo } \\
\text { tiempo-a determinadas estructuras } \\
\text { dominantes. } \\
\text { Es un esquema que no condiciona a priori la } \\
\text { acción particular del líder }\end{array}$ \\
\hline $\begin{array}{l}\text { Proceso de } \\
\text { actuación }\end{array}$ & $\begin{array}{l}\text { Por estandarización. Los métodos de } \\
\text { actuación se encuentran diseñados y } \\
\text { son implementados para responder - } \\
\text { ajustar- a los marcos de actuación }\end{array}$ & $\begin{array}{l}\text { Por pragmatismo. Se basa en la acción - } \\
\text { solución- que funciona para un contexto } \\
\text { particular. La evaluación de la acción no se } \\
\text { realiza comparando con marcos de actuación } \\
\text { —-generales y previos—, sino que se centra } \\
\text { en la propia acción práctica y particular. El } \\
\text { control es ad-hoc y a posteriori de la acción } \\
\text { —sin deducción desde lo general previo-. } \\
\text { El control supone evaluar lo particular con } \\
\text { criterios internos y coyunturales, y supone } \\
\text { evaluar la articulación de lo particular con } \\
\text { lo general (que no es pre-existente y es } \\
\text { dinámico) }\end{array}$ \\
\hline Lógica de solución & $\begin{array}{l}\text { Lineal. Con una episteme argumentativa } \\
\text { de tipo problema-solución. El problema } \\
\text { aparece como elemento objetivo (una } \\
\text { falla del marco) y la solución como } \\
\text { ejecutiva, para responder a la falla y } \\
\text { ajustar el marco }\end{array}$ & $\begin{array}{l}\text { No lineal. La voluntad del liderazgo actúa } \\
\text { invirtiendo la racionalidad con un tipo de } \\
\text { episteme argumentativa solución-problema. } \\
\text { El problema es una justificación a posteriori }\end{array}$ \\
\hline
\end{tabular}


Horizonte temporal de la acción
Largo plazo. La estabilidad del marco de actuación es la base de la cooperación en la democracia pluralista. También, la estabilidad es la base de la sinopsis en la lógica lineal. El cambio es solamente incremental-instrumental, para ajustar el marco de actuación
Corto plazo. Es un esquema muy dinámico. Se trata de un cambio incremental e innovador, ya que se rescata del pasado la diferencia del proceso dialéctico no lo común o universal de un marco de actuación-y ciertas estructuras dominantes por decisiones estratégicas de deconstrucción

Fuente: Elaboración propia.

Como pudo observarse, la racionalidad política y su impacto en la argumentación es completamente la opuesta. En Occidente, la horizontalidad democrática otorga flexibilidad argumentativa y los marcos de actuación estables la restringen (North, 1993; Olson, 1993). En China, el dinamismo institucional otorga flexibilidad argumentativa y el orden jerárquico y particular la restringe ${ }^{4}$. Democracia con estabilidad versus jerarquía con cambio (Corkin, 20l4), ambos esquemas políticos estableciendo los límites argumentativos ${ }^{5}$.

En este contexto, el enfoque de agenda (Cohen, 1993) nos permite considerar las distintas dimensiones de la racionalidad y retórica política china: la jerarquía establecida en un texto; la no-linealidad de la agenda de soluciones; los tipos de marcos habilitantes, contradictorios y estratégicos; el contexto particular y su relación con lo general-dado; y finalmente, el dinamismo del contenido de la información.

La explicitación frecuencial y de relacionamiento de temas y atributos (Winter y Eyal, I 98I; McCombs y Evatt, 1995) de la agenda —aplicado al discurso político chino- sostiene un margen de libertad de argumentación en la audiencia (cuadros situados), para que este destinatario de la agenda aporte el componente diferenciador y produzca una novedosa síntesis dialéctica en el contexto particular (Xi, 20l4). Es decir, la lógica no lineal, centrada en la voluntad —solucióndel líder (Schopenhauer, 2005) es el motor del cambio chino, ya que incentiva pero no limita. Luego, el actor situado produce el desarrollo del argumento completo, con la identificación de las condiciones de factibilidad y del propio problema a resolver. Entonces, el enfoque de agenda capta esta esencia de racionalidad política en China.

En Occidente, los estudios de agenda no siguen este eje. La agenda condiciona (Fishman, 1983) en lugar de habilitar a la audiencia, debido a la propia lógica restrictiva de los marcos de actuación. Además, el componente particular (datos situados) legitima lo general por procesos deductivos e inductivos (cumpliendo una función secundaria ${ }^{6}$ ), mientras que en la retórica política china lo particular materializa la toma de decisión y la síntesis dialéctica ${ }^{7}$.

4 Como ejemplo, la libertad de mercado es controlada a posteriori y jerárquicamente en China (economía de mercado socialista), a diferencia del modelo occidental que define democráticamente y a priori los marcos de actuación que limitan dicha libertad.

5 Es importante destacar que así como la jerarquía tiene una connotación negativa desde la perspectiva democrática, lo mismo sucede con la estabilidad para la filosofía china (Wright, 1953).

6 En realidad, lo particular es siempre excepción de lo general y lo dado, aunque cumpla una función de legitimación en el argumento formal de Occidente.

Al ser nuestro objeto el discurso político chino, la agenda no condiciona. Sin embargo, lo particular representa un mayor nivel de orientación de la agenda sobre la audiencia. 
De esta forma, el presente trabajo propone un aporte original - teórico y de aplicación empírica - en el campo de los estudios sociales y políticos de la agenda como en Wang (2016), al adaptar los mismos a la racionalidad política china.A continuación, se profundiza en esta adpatación desde los requisitos metodológicos.

\section{Metodología}

El estudio del discurso (Bach, 200I;Van Leeuwen, 2008) y, en particular, el análisis de contenido (Kolbe y Burnett, I99 I; Krippendorff, 1990) ha tenido una gran diversidad de variantes de aplicación (Sanchez, 20I I). En nuestro caso, adaptamos los estudios de agenda (Aruguete, 20I5) a un original objeto de investigación para la identificación de contenido, sus relaciones y representatividad en el discurso político chino.

La agenda "no nos dice 'qué pensar' sino que influye sobre la audiencia en 'sobre qué pensar'" (Cohen, 1993, p. 13). De forma operativa, la agenda se produce por la frecuencia de aparición de ciertos términos clave y por el relacionamiento espacial de estos términos. Un término con alta frecuencia pero no relacionado con otros términos de alta frecuencia no es relevante en este tipo de análisis ${ }^{8}$. Esto último se debe a que las audiencias acceden de forma intuitiva a los términos asociados, lo cual se vincula con el sesgo de confirmación de la audiencia (D’Adamo, García Beaudoux y Freidenberg, 2007; Festinger, 1957). Las agendas cristalizan contenidos ya presentes en la memoria de la audiencia y esto es posible con activaciones de términos en tándem (Bantimaroudis, Ban y Kiousis, 1999).

Por este motivo, nuestra metodología se centra en los sustantivos comunes (se utilizan técnicas Part of Speech Tagging y procesos de lematización), su frecuencia, y en su relacionamiento por concordancia — vecindad-, y evita considerar a los adjetivos —atributos afectivos-, así como a los verbos y a otros tipos de relacionamientos gramaticales que indican el qué pensar. Se trata de una agenda que evita la formalidad del argumento y adopta un enfoque pragmático (Belinchón Carmona, 1984). En este sentido, se utiliza el método KWIK (Key Word in Context), y no el de co-ocurrencia, dado que KWIC no reconoce la regla sintáctica de puntuación y permite enfocarnos en las relaciones espaciales.

En el presente trabajo se construye una lista de términos clave que alcanza el $40 \%$ de la distribución superior de frecuencia (Kiousis, 2004). Esto acota el análisis a un determinado nivel de relevancia de la agenda. Luego, se elaboran matrices simétricas de concordancia. Es decir, en el contexto de vecindad de los términos clave se identifican otros términos de alta frecuencia —dentro del $40 \%$ de distribución superior-, incluyendo a los nombres propios (una entidad de alta frecuencia es, en primer lugar, parte del enfoque de agenda y no cumple la función de ser excepción particular).

También, nuestra investigación identifica los recursos de autoridad (su diversidad y

De todas formas, en el discurso político chino, cuanto mayor es la frecuencia absoluta mayores son las posibilidades de encontrar términos de alta frecuencia en un contexto de vecindad. 
prestigio ${ }^{9}$ ) que materializan la particularización sobre los temas y sus atributos. Consideramos dos recursos centrales de autoridad para esta tarea: los datos y los nombres propios de actores e instituciones ${ }^{10}$. En todo proceso comunicativo estos dos recursos potencian lo particular y se distancian de lo dado y general, que no requiere de mayor autoridad u orientación. Esto significa que cada término clave se pondera de acuerdo a la presencia —en su vecindad- de recursos de autoridad. La cantidad relativa de recursos de autoridad (recursos de autoridad/frecuencia léxica) determina el tamaño de los nodos en la representación espacial de este trabajo.

Finalmente, los términos y, por lo tanto, sus relaciones y recursos asociados son sometidos a un análisis del índice de concentración Herfindahl-Hirschman ( $\left.\mathrm{HH}^{\prime \prime}\right)$.

De esta forma, nuestra metodología permite identificar temas y atributos dados — sin recursos de autoridad - como marcos habilitantes, contradictorios y deconstructivos que indican un camino de soluciones.Y, por otra parte, se obtienen temas y atributos particulares -asociados a recursos de autoridad- Son de interés los límites entre lo particular y lo dado, ya que un contenido semántico de este tipo refleja los puentes entre el marco y su materialización particular.

En definitiva, la agenda china se presenta como una metaestructura para la argumentación, con soluciones y actores e indicios de factibilidades. Se trata de un enfoque pragmático - no formal- donde la creencia en la agenda habilita a la argumentación situada. A continuación, representamos en el cuadro 2 los tipos discursivos posibles según nuestro marco teórico y metodológico.

\section{Cuadro 2. Tipos discursivos según marco y recursos}

\begin{tabular}{|c|c|c|}
\hline \multirow{2}{*}{ Marco discursivo } & \multicolumn{2}{|c|}{ Recursos de autoridad } \\
\cline { 2 - 3 } & No & $\mathrm{Si}$ \\
\hline Occidental & Discurso normativo & Discurso científico \\
\hline Chino & Discurso político & Discurso particularista \\
\hline
\end{tabular}

Fuente: Elaboración propia.

Dado el marco teórico y metodológico explicitado, se justifica la formulación de nuestra hipótesis de investigación. Es decir, las categorías descritas (agenda política, particularizaciones y sus combinaciones) son útiles para la identificación de tendencias en un análisis evolutivo del discurso político chino.

Por último, cabe mencionar que utilizamos para la representación de los resultados

9 Los enfoques pragmáticos del uso del lenguaje se centran en interpretar la selección de las fuentes (Belinchón Carmona, 1984).

10 Los recursos como datos pueden referenciar al número de una Ley, fechas o estadísticas. Los actores e instituciones son casos y ejemplos particulares, incluidos los lugares. Técnicas como Named Entity Recognition se utilizaron en la identificación automática (se utilizó Amazon Comprehend y el software GATE de la Universidad de Sheffield para esta tarea).

" El HHI (Herfindahl-Hirschman Index) nos permite identificar la dispersión de los términos — sus relaciones y recursos- en secciones (las divisiones estructurales del corpus). Una agenda específica puede concentrarse en cierto momento del texto y, por lo tanto, puede indicar la importancia de esa sección. 
algoritmos de espacialización ${ }^{12}$. En dicha representación, la fuerza del relacionamiento -que indirectamente considera la frecuencia absoluta - es la que define la dispersión de los términos. Esto nos permite realizar operaciones propias de la matemática de vectores (Vylomova, Rimell, Cohn y Baldwin, 2016). Por su parte, el tamaño de los nodos se encuentra definido por la cantidad de recursos de autoridad asociados a los términos y el color de los nodos representa el nivel de dispersión o concentración en secciones del corpus (dado el valor de control del HHI).

\section{Resultados}

Los dos documentos analizados son versiones de la perspectiva oficial que la República Popular China busca comunicar —en idioma inglés - al resto del mundo. Este tipo de documento de la más alta jerarquía (White paper) es preparado con mucho detalle por el Gobierno central chino y de aquí surge su representatividad como objeto de estudio para esta investigación. The Facts and China's Position on China-US Trade Friction (Los Hechos y la Posición de China en la Fricción Comercial China-Estados Unidos), de septiembre de 2018, posee unos 20.795 tokens en setenta y un páginas. Por su parte, el documento China's Position on the China-US Economic andTrade Consultations (La Posición de China en las Consultas Comerciales y Económicas China-Estados Unidos), de junio de 2019, posee 5.448 tokens en diecinueve páginas.

Es importante comentar que la metodología descrita anteriormente se aplicó de forma rigurosa en todas sus fases. Sin embargo, la propia retórica política china obligó a considerar ciertos adjetivos y unidades de medida como términos clave de la agenda. En el primer caso, por tratarse de elementos que conforman términos compuestos, que son típicos del discurso político chino. En el segundo caso, para destacar recursos de autoridad que son parte del $40 \%$ de la distribución superior de la agenda.

Finalmente, otra cuestión que merece aclaración es que el corpus de cada documento fue dividido en sus secciones estructurales (subtítulos, sobre todo): catorce secciones en el documento The Facts and China's Position on China-US Trade Friction y dieciocho en el documento China's Position on the China-US Economic and Trade Consultations. En este contexto, el HHI controla que los términos que se encuentren excesivamente concentrados en determinadas secciones no se presenten como representativos de todo el corpus.

A continuación, presentamos el resultado gráfico de la aplicación de la metodología sobre el objeto de estudio y, luego, el análisis estático y dinámico.

12 El algoritmo Yifan-Hu (20I2) que utilizamos trabaja la fuerza de repulsión agrupando nodos (clusters), lo cual resulta importante para representar marcos de una agenda. 


\section{Gráfico I. Identificación de agenda, recursos de autoridad y HHI sobre los documentos}

The Facts and China's Position on China-US Trade Friction (izquierda) y China's Position on the China-US Economic and Trade Consultations (derecha)
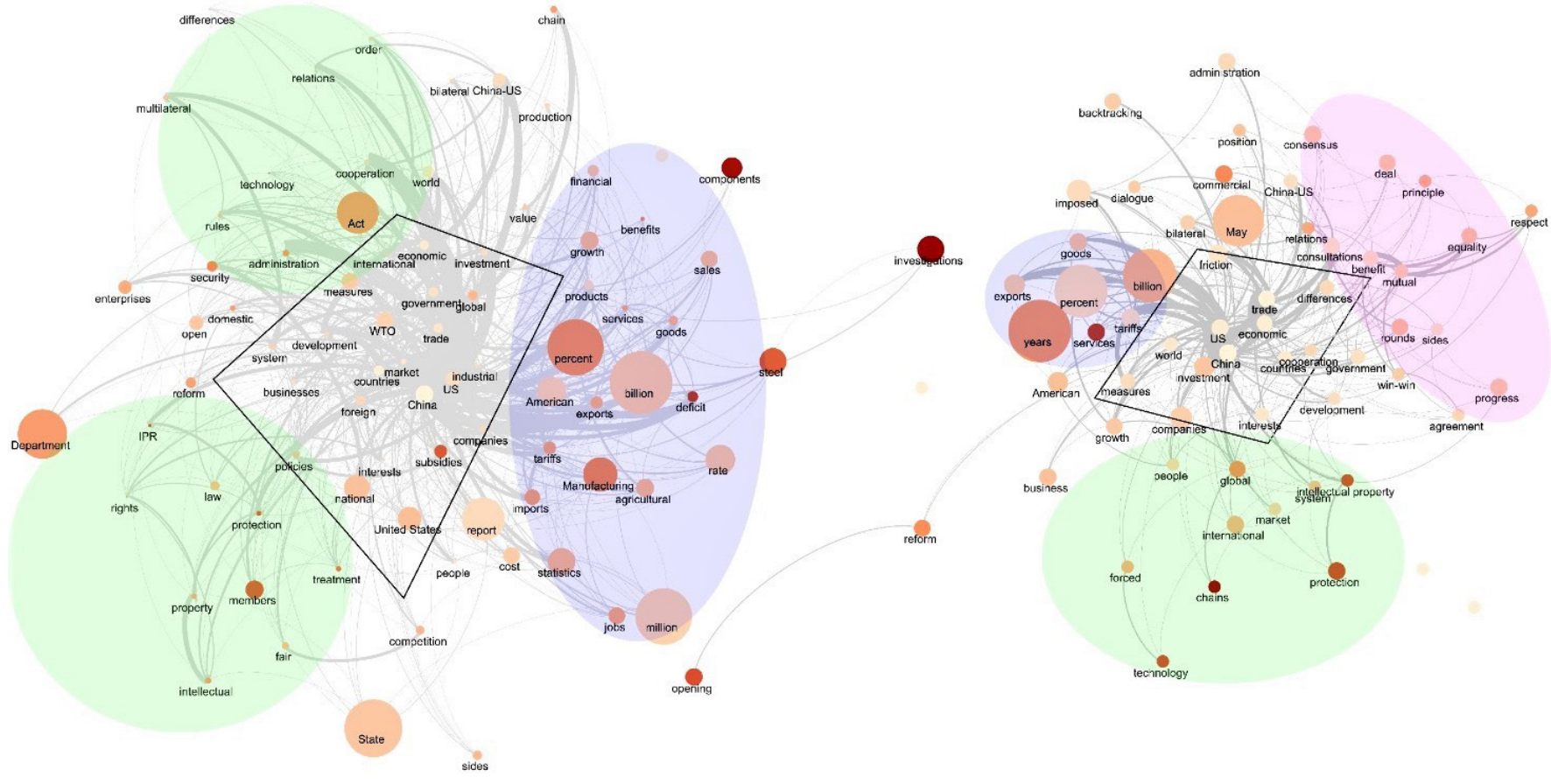

Fuente: Elaboración propia.

\section{I Análisis estático}

Respecto al documento The Facts and China's Position on China-US Trade Friction (en la izquierda del gráfico I) destacamos que los elementos de mayor centralidad — dentro del polígono central de este documento- referencian, en primer orden, la relación comercial entre China y Estados Unidos, ya que es sobre este tema principal de lo que trata todo el documento. Sin embargo, hay otros dos elementos centrales de mucha relevancia. Uno, el actor Organización Mundial de Comercio (OMC) como recurso de autoridad que particulariza las posiciones del discurso chino. Dos, el sector industrial que permite agrupar una serie de atributos, como las cadenas que se afectan con la fricción entre China y Estados Unidos. En las áreas más externas del polígono central encontramos el tema inversiones y todas las acciones (medidas y políticas proteccionistas, así como subsidios ${ }^{13}$ ) que se relacionan con intereses nacionales y que afectan a la comunidad global. Esta es la agenda central del documento de septiembre de 2018.

Luego, encontramos tres gandes marcos en este mismo documento. Un primer marco (círculo verde inferior) con el debate sobre la propiedad intelectual en China. Un segundo marco (círculo verde superior) con las reglas y normas pre-existentes para la cooperación, entre ellas las formas pre-establecidas de transferencia tecnológica. Haciendo de puente entre ambos marcos

13 La mención a los subsidios se encuentra muy concentrada en el apartado tercero del documento. 
encontramos el concepto de reforma y apertura ${ }^{14}$, que nos viene a recordar el espíritu con el cual deben interpretarse dichos marcos desde la posición de China. El tercer marco es el de mayor recursos de autoridad (círculo azul) y, de hecho, es el marco con mayor superficie de los tres. Esto indica que el documento contiene altos niveles de datos y nombres propios para particularizar el discurso. Este tercer marco trata de los bienes y servicios producidos y exportados e importados en la relación China-Estados Unidos. Es muy importante mencionar que, en este tercer marco, se plantean los niveles de tarifas y que se mencionan sectores concretos con alta frecuencia y relacionamientos: el sector financiero y agrícola y la industria del acero.

En el documento China's Position on the China-US Economic and Trade Consultations (en la derecha del gráfico I) los elementos de mayor centralidad son la relación comercial y económica entre China y Estados Unidos. También se destaca — dentro del polígono central- el tema inversiones y la cooperación, así como las medidas que responden a los intereses nacionales y que tienen como resultado diferencias y fricción que afectan a la comunidad global. Este es el eje central de la agenda en este documento de junio de 2019.

Luego, hay otros tres marcos de interés en este segundo documento. Primero, el debate sobre la propiedad intelectual y la transferencia tecnológica en China (círculo verde inferior). Segundo, un marco donde los recursos de autoridad particularizan a los bienes y servicios, sus niveles tarifarios y comerciales (círculo azul). Tercero, se presenta un marco con términos típicos de la retórica política china (círculo violeta), que refiere a valores, principios y formas de actuación para fomentar la cooperación. Entre el marco segundo y el tercero actuá como puente el mes de mayo, que vine a representar el período temporal de mayor fricción $y$, al mismo tiempo, de consultas entre China y Estados Unidos. Decimos que actúa como un puente ya que parece transformar los retrocesos y las acciones impuestas en una oportunidad para aplicar los principios de la cooperación (el círculo violeta).

\subsection{Análisis dinámico}

Entre septiembre de 2018 y junio de 2019 podemos afirmar que existen continuidades en la retórica de los documentos oficiales chinos sobre la guerra comercial entre China y Estados Unidos. El eje central de la agenda busca, en ambos casos, expandir el debate comercial entre China y Estados Unidos, transformándolo en una discusión sobre distintos aspectos económicos: las cadenas industriales y las inversiones. Un segundo objetivo de ambos documentos es contraponer la diferencia entre los intereses nacionales y la comunidad internacional de países. También, en los dos documentos, la utilización de recursos de autoridad sobre los niveles comerciales y tarifarios da soporte al eje central ya mencionado. Otra continuidad —aunque no tan central- es el debate sobre la propiedad intelectual y transferencia tecnológica en China. Estas tendencias son muy contundentes en cuanto a frecuencias y relacionamientos $y$, por lo tanto, sin duda representan muy bien la posición de la agenda de China a lo largo del tiempo.

Respecto a las discontinuidades son tres, y muy marcadas también. Primero, el documento de 2019 centraliza la cooperación, cuestión que no se ve reflejada en el documento de 2018 .

\footnotetext{
14 Se trata de una utilización metafórica (Musolff, 2004) de las reformas de diciembre de 1978, que permitieron iniciar una nueva era de vinculación exterior de China.
} 
Segundo, la superficie que abarca la particularización discursiva en el documento de 2018 es mucho más amplia que en el documento de 2019. Finalmente, y tercero, el segundo documento incluye un marco de principios y valores para la cooperación en reemplazo de un marco —en el documento de 2018- mucho más legalista y de retórica normativa occidental.

\section{Conclusión}

Hemos podido demostrar el cumplimiento de nuestra hipótesis de investigación, ya que en los documentos oficiales chinos sobre la guerra comercial con los Estados Unidos existen tendencias sobre la agenda política, los recursos particularistas utilizados y la articulación de ambos.

El documento The Facts and China's Position on China-US Trade Friction amplía la discusión comercial hacia las cadenas industriales y las inversiones. Se enumeran las medidas que se corresponden con los intereses nacionales y que afectan a la comunidad internacional, particularizando esto último en la arena de la OMC. También se particulariza con datos y nombres propios a los bienes y servicios del comercio y a las tarifas que se aplican. Esto último da soporte a los elementos centrales de la agenda. Otros dos marcos son relevantes: la discusión sobre propiedad intelectual y las menciones de reglas y normas para fomentar la cooperación. Este segundo marco - bastante restringido - actúa como marco habilitante, aunque omite la retórica china de valores y principios, acercándose bastante a un discurso normativo occidental.

El documento China's Position on the China-US Economic and Trade Consultations mantiene la tendencia del eje central del documento de 2018 , pero es menos agresivo, al centralizar a la cooperación como objetivo y al no recurrir a particularizaciones. Tampoco ofrece un soporte tan importante de recursos de autoridad como lo hace el documento de 2018. Además, se mantiene en el documento de 2019 la tendencia del debate sobre la propiedad intelectual en China como algo muy importante. Sin embargo, como cambio relevante, este segundo documento presenta una agenda política mucho más cercana a los marcos contradictorios y dialécticos de la clásica retórica confuciana y marxista de China (se utilizan términos como beneficio mutuo, respeto mutuo o relaciones ganar-ganar, entre otros).

Consideramos, por lo tanto, que hay una tendencia marcada y coincidencias importantes (expandir la discusión desde lo comercial a lo económico, criticar al proteccionismo y sostener una posición en el debate sobre la propiedad intelectual en China) pero también las diferencias son relevantes. En especial, cómo el segundo documento abarca de forma más amigable la retórica política china de valores y principios cooperativos y prescinde de los recursos de autoridad. En este sentido, recordemos que una menor particularización implica menores niveles de orientación.

Desde el punto de vista de nuestro marco teórico, ambos documentos buscan destacar la eficacia colectiva (el desarrollo de la comunidad global) y trabajan sobré lógicas de soluciónproblema. Además, el cambio entre los dos documentos refleja el dinamismo en la utilización de recursos discursivos por parte de China. En definitiva, el documento de 2019 tiene mayores características de discurso político chino que incentiva al pragmatismo situado y que no 
condiciona ${ }^{15}$, mientras que el documento de 2018 desarrolla una posición más deconstructiva sobre la retórica occidental del conflicto comercial, incluso utilizando herramientas que son más propias del discurso científico (con deducciones o inducciones desde los recursos de autoridad).

El discurso político chino es dinámico, se adapta al contexto con una multiplicidad de formas posibles. Identificar continuidades y cambios de este discurso desde la comprensión teórica de su racionalidad política resulta fundamental para interpretar los conflictos actuales, como lo es la guerra comercial con los Estados Unidos.

\section{Referencias}

Aruguete, N. (20I5). El Poder de la Agenda. Política, Medios y Público. Biblos.

Bach, K. (200I).Semantically Speaking. En Kiefer, F.; Kenesei, I.y Harnish, R. M. (Eds.). Perspectives on Semantics, Pragmatics, and Discourse (pp. 147-I70). John Benjamins.

Bantimaroudis, P., Ban, H. y Kiousis, S. (1999). Candidate Image Attributes: Experiments on the Substantive Dimension of Second Level Agenda Setting. Comunication Research, 4 (26), 4/4-428. https://doi.org// 0.I I 77\% 2F009365099026004003

Belinchón Carmona, M. (1984). Adquisition and evaluation of pragmatic functions of language: A developmental study. Studies in Psychology, 19 (5), 35-49. https://doi.org/ I 0. I080/02 I09395. 1984. I082 I 400

Bell, D. (2008). China's New Confucianism. Politics and Everyday Life in a Changing Society. Princeton University Press.

Callahan,W. (2016). China's Asia dream:The Belt Road initiative and the new regional order. Asian Journal of Comparative Politics, I (3), 226-243.

Chase, M. (20I2). China's search for a "New Type of Great Power Relationship". China Brief, I7 (I2), I2- 16.

Cheng, C. (1991). New Dimensions of Confucian and Neo-Confucian Philosophy. State University of New York.

Cohen, B. (1993). The Press and Foreign Policy. University of California.

Corkin, L. J. (20|4). China's Rising Soft Power: the role of Rhetoric in constructing China-Africa relations. Revista Brasileira de Política Internacional, 57, 49-72. http://dx.doi.org/I0.I590/0034-732920 I 400204

Connelly, J. M. (199I). China: de la diplomacia revolucionaria a la diplomacia de la paz y el desarrollo. Estudios de Asia y Africa, 2 (26), 296-3I4.

Coulthard, M. (1996). On Analysing and Evaluating Written Text. En Coulthard, M. (Ed.). Advances in Written Text Analysis (pp. 219-228). Routledge.

D’adamo, O., García Beaudoux,V.y Freidenberg, F. (2007). Medios de comunicación y opinión pública. Mc Graw Hill.

Eyal, C. H. (1980). Time Frame in Agenda Setting Research:A study of the conceptual and methodological factors affecting the time frame context of the agenda setting process. Syracuse University.

Fairclough, N. (2003). Analysing Discourse:Textual Analysis for Social Research. Routledge.

Festinger, L. (1957). A theory of cognitive dissonance. Stanford University Press.

Fishman, M. (1983). La fabricación de la noticia. Tres Tiempos.

Gou, L. (20I4). Toward the third level of agenda setting theory. En Johnson, T.J. (Ed.). Agenda setting in a 2.0 World (PP. I | 2-I33). Routledge.

Hu, Y. (marzo, 20I2). A maxent-stress model for graph layout. Proceedings de IEEE Pacific Visualization Symposium, Songdo.

Kiousis, S. (2004). Explicating Media Salience: A Factor Analysis of New York Times Issue Coverage During the 2000 U.S. Presidential Election. Journal of Communication, I (54), 7I-87.

Kolbe, R.y Burnett, M. (1991). ContentAnalysis Research:An Examination of Applications with Directives for Improving Research Reliability and Objectivity. Journal of Consumer Research, 2 (I8), 243-250.

Krippendorff, K. (1990). Metodología de Análisis de Contenido. Teoría y Práctica. Paidós Comunicación.

Leonard, M. (2008). What does China thinks? Public Affaires.

$\mathrm{Li}, \mathrm{C}$. (2006). The Confucian Ideal of Harmony. Philosophy East and West, 4 (56), 583-603. Recuperado de: http://www. jstor.org/stable/4488054 (0I.07.2020).

$\mathrm{Li}, \mathrm{C}$. (20I4). The Confucian Philosophy of Harmony. Routledge.

Li,W. (2019). Towards Economic Decoupling? Mapping Chinese Discourse on the China-US Trade War. The Chinese Journal of International Politics, 5 19-556.

Lima Bandeira, M. y Rabaii, N. (2016). El valor del análisis de discurso en los estudios comparativos de políticas públicas. Estudios Políticos, 49, 38-67.

\footnotetext{
15 En el documento de 2019 la particularización se relaciona de forma más directa con nuestro marco teórico sobre la racionalidad política china, ya que se destaca la particularización temporal (el mes de mayo, por ejemplo).
} 
McCombs, M. y Evatt, D. (1995). Los temas y los aspectos: explorando una nueva dimensión de la agenda setting. Comunicación y Sociedad, I (8), 7-32.

Moncada Durruti, M. (20I I). El despertar del neoconfucianismo en China. Impacto en el discurso político del Partido Comunista Chino. Memoria y Civilización, I4, 20I-22I.

Musolff, A. (2004). Metaphor and Political Discourse, Basingstoke. Palgrave Macmillan.

North, D. (1993). Instituciones, Cambio Institucional y Desempeño Económico. Fondo de Cultura Económica.

Oficina de Información del Consejo de Estado de la República Popular China (2018). The Facts and China's Position on China-US Trade Friction (Los Hechos y la Posición de China en la Fricción Comercial China-Estados Unidos), septiembre de 2018. Beijing: SCIOPRC.

Oficina de Información del Consejo de Estado de la República Popular China (2019). China's Position on the China-US Economic and Trade Consultations (La Posición de China en las Consultas Comerciales y Económicas ChinaEstados Unidos), junio de 2019. SCIOPRC.

Olson, M. (1993). Dictatorship, Democracy, and Development. American Political Science Review, 3 (87), 567-576.

Oviedo, E. D. (noviembre, 20 14). Idioma y poder. Conceptos clave del discurso político chino en sus relaciones con América Latina. Actas del Primer Congreso Internacional de Sinología en Español,Taipei.

Rocha Pino, M. de J. (20I8). El discurso de China sobre el Nuevo Modelo de Relaciones entre Grandes Potencias y la relación con Estados Unidos durante los gobiernos de Bush y Obama (2005-2017). Revista Mexicana de Ciencias Políticas y Sociales, 233 (63), 193-220.

Sanchez, L. (20I I). Elementos para el análisis del discurso político en el escenario internacional. Revista Internacional de Pensamiento Político, I (6), 4I9-433.

Schopenhauer, A. (2005). El mundo como voluntad y representación. Akal.

Tao,W. (2009). The Financial Crisis and Sino-US Relations. Peace and Development, 4, 28-40.

Van Leeuwen, T. (2008). Discourse and Practice: New Tools for Critical Discourse Analysis. Oxford University Press.

Vylomova, E., Rimell, L., Cohn,T.y Baldwin, T. (2016). Take and Took, Gaggle and Goose, Book and Read: Evaluating the Utility of Vector Differences for Lexical Relation Learning. Working Paper Series Cornell University, I 509.01692, I - I 2. Recuperado de: https://arxiv.org/abs/I509.0I692 (05.07.2020).

Wang, F. (2008). Competitive Interdependence Between China and the US. World Economics and Politics, 3, I9-42.

Wang, J. (2016). New political and communication agenda for political discourse analysis: Critical reflections on critical discourse analysis and political discourse analysis. International Journal of Communication, 10, 2766-2784.

Wang, J. (2017). Representations of the Chinese Communist Party's political ideologies in President Xi Jinping's discourse. Discourse \& Society, 4 (28), 4I 3-435. https://doi.org/ I 0. I I 77\%2F09579265 I 66874 I 8

Winter, J. y Eyal, C. (198I). Agenda Setting for the Civil Right Issue. Public Opinion Quarterly, 3 (45), 376-383.

Wodak, R. (2009). The Discourse of Politics in Action: Politics as Usual. Palgrave Macmillan.

Wright, A. (1953). Struggle vs. Harmony: Symbols of Competing Values in Modern China. World Politics, I (6), 3 I -44.

Wu, X. (20I4). Agenda for a New Great Power Relationship. The Washington Quarterly, I (37), 65-78.

$\mathrm{Xi}$, J. (20I4). La gobernación y administración de China. China, Ediciones en Lenguas Extranjeras Compañia Limitada.

Xing, G. (2009). Hu Jintao's political thinking and legitimacy building: A post-Marxist perspective. Asian Affairs: An American Review, 4 (36), 2 I 3-226.

Xu, J. (2018). The Adjustment of US Policy Towards China and Three Major Risks in China-US Relations. China International Studies, 4, I4-28.

Xu, S. (20I4). Chinese Discourse Studies. Palgrave Macmillan.

Yuan, P. (2010). Whither Are Sino-US Relations Going? Foreign Affairs Review, 2, 2-17.

Zeng, J.y Breslin, S. (2016). China's 'New Type of Great Power Relations': a G2 with Chinese characteristics. International Affairs, 4 (92), 773-794.

Zhao, K. (20I3). Sino-US Relations in the Post-Financial Crisis Era. The Chinese Journal of American Studies, I, 48-6I.

Zhao, M. (2019). Is a New Cold War Inevitable? Chinese Perspectives on US-China Strategic Competition. The Chinese Journal of International Politics, 2019, 37I-394. 


\section{RELACIONES INTERNACIONALES}

Revista académica cuatrimestral de publicación electrónica Grupo de Estudios de Relaciones Internacionales (GERI)

Universidad Autónoma de Madrid, España

https://revistas.uam.es/relacionesinternacionales

ISSN 1699 - 3950

f facebook.com/RelacionesInternacionales

3. twitter.com/RRInternacional

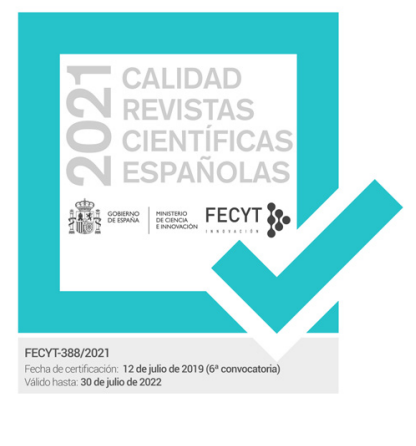

